\title{
Determination of the Spin-Rotation and Spin-Spin Interaction Constants of Germane by Microwave Fouriertransform Measurements
}

\author{
Wolfgang Stahl and Helmut Dreizler \\ Abteilung Chemische Physik im Institut für Physikalische Chemie der Universität Kiel \\ Z. Naturforsch. 42 a, $1402-1404$ (1987); received September 29, 1987 \\ We determined the tensorial spin-rotation- $\left(c_{d}\right)$ and the spin-spin interaction constant $(d)$ \\ of germane from the hyperfine splittings of Q-branch microwave lines measured by microwave \\ FOURIER transform (MWFT) spectroscopy.
}

\section{Introduction}

In a previous paper [1] we reported on the investigation of the vibrational ground state microwave spectrum of the isotopically enriched germane species ${ }^{70} \mathrm{GeH}_{4},{ }^{72} \mathrm{GeH}_{4}$ and ${ }^{74} \mathrm{GeH}_{4}$. We pointed out that most of the F-type lines were broadened or split due to spin-rotation and spin-spin interaction. Three examples are given in Figure 1.

The magnitude of the splitting depends on the respective transition and ranges up to $100 \mathrm{kHz}$. As expected an isotopic dependence was not observed. In many cases the hyperfine structure could not be completely resolved. To increase the precision of the frequencies of the multiplet components we performed a line shape analysis [2]. These data provided the basis of the fit of the tensorial spin-rotation constant $c_{d}$ and the spin-spin interaction constant $d$.

\section{Theory}

There exist many theoretical contributions in the field of spin-rotation and spin-spin interaction of tetrahedral molecules [3-9]. We will not repeat either of them. We based our calculations on the formulas given by Hougen [7] because they seam to be most convenient in practical application. The hyperfine energies of the $F_{1}$ and $F_{2}$ species ground state transi-

Reprint requests to Prof. Dr. H. Dreizler, Abteilung Chemische Physik im Institut für Physikalische Chemie, ChristianAlbrechts-Universität, Olshausenstr. 40-60, D-2300 Kiel 1 , FRG. tions are given by Eq. (16) of [7],

$$
\begin{aligned}
E & =h_{0} D_{t}+\left[-c_{a}+h_{1} c_{d}\right](C / 2) \\
& +h_{2} d[(3 / 4) C(C+1)-I(I+1) J(J+1)] / J(J+1) .
\end{aligned}
$$

$h_{0}, h_{1}$ and $h_{2}$ are numerical values depending on the respective transition. $h_{0}$ is tabulated in [9] (there it is called $f$ ), $h_{1}$ and $h_{2}$ are found in [7]. $D_{t}$ is the tensorial centrifugal distortion constant, $c_{a}$ represents the scalar spin-rotation constant. $C$ ist defined by

$$
C=F(F+1)-J(J+1)-I(I+1),
$$

where $J$ is the quantum number of the overall rotational momentum operator $\hat{J}, I$ the quantum number of the coupled four proton spin operators $\hat{I}$ and $F$ the quantum number of the total angular momentum operator $\hat{F}$ composed of $\hat{J}$ and $\hat{I}$. For the case of $F$-type transitions $I$ can be shown to be always equal 2. For Q-branch transitions predominantly $\Delta F=0$-lines are observed. In this case $\Delta C=0$ and the transition frequency is proportional to

$$
\begin{aligned}
\Delta E= & \left(h_{0}^{\mathrm{u}}-h_{0}^{1}\right) D_{t}+\left(h_{1}^{\mathrm{u}}-h_{1}^{1}\right) c_{d}(C / 2) \\
& +\left(h_{2}^{\mathrm{u}}-h_{2}^{1}\right) d[(3 / 4) C(C+1) \\
& -I(I+1) J(J+1)] /[J(J+1)] .
\end{aligned}
$$

The superscripts $u$ and 1 indicate the upper and the lower level. Obviously the scalar spin-rotation constant $c_{a}$ is not determinable.

The splitting between two lines with different $F$ quantum numbers (indicated with ' and ") of a hyperfine triplet can be expressed as

$$
\Delta(\Delta E)=A c_{d}+B d
$$

0932-0784 / 87 / 1200-1402 \$01.30/0. - Please order a reprint rather than making your own copy. 


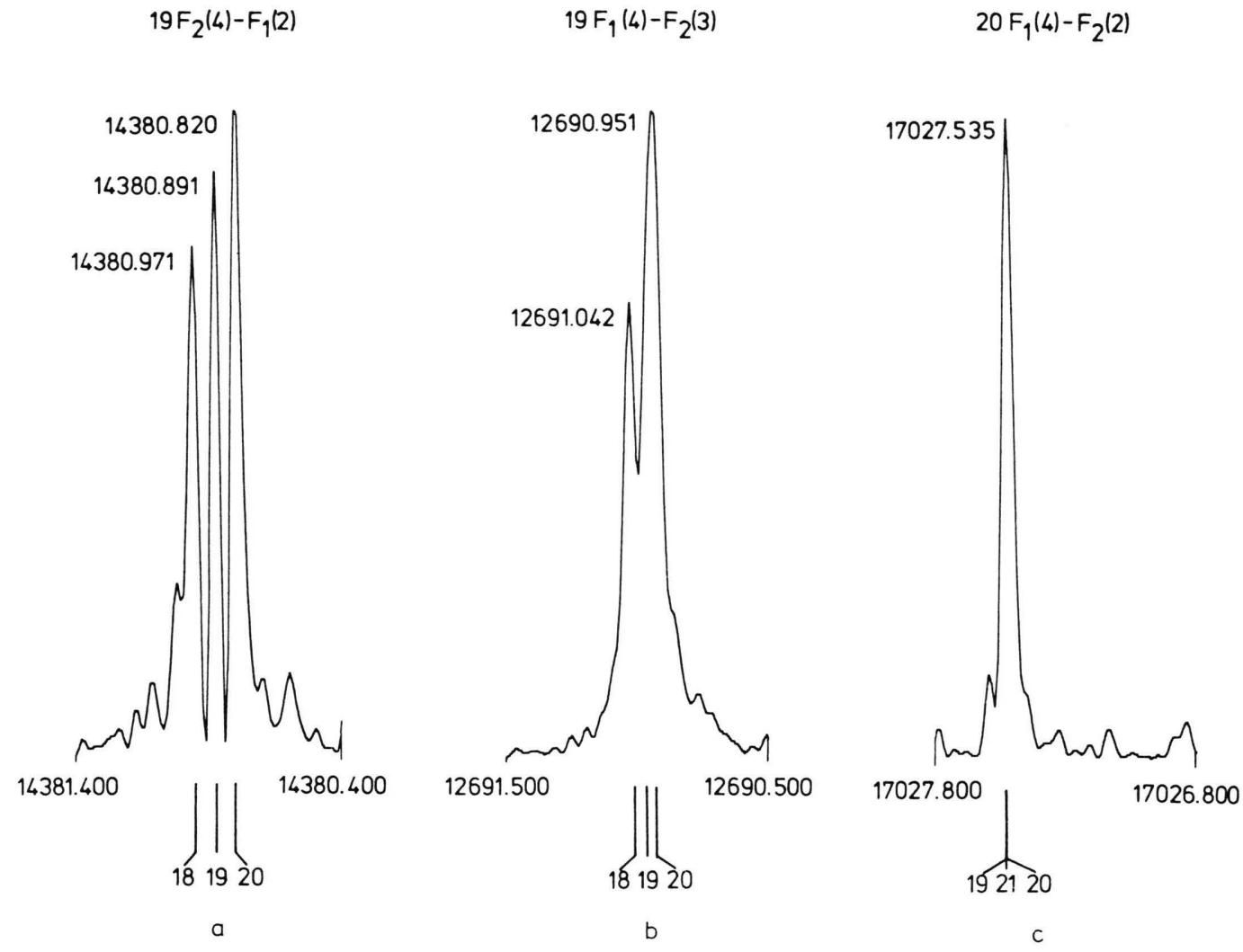

Fig. 1. Three examples for $F$-type transitions. Measurements and theoretical patterns (calculated with parameters of Table 1 fitted to transitions in Fig. 1 a) are shown. Frequencies in $\mathrm{MHz}$. Hyperfine components are labelled with $F$-quantum numbers. a) Rotational transition $19 F_{2}(4)-F_{1}(2)$ of ${ }^{74} \mathrm{GeH}_{4}$. Cell temperature: $20^{\circ} \mathrm{C}$. Gas pressure: $3.8 \mathrm{mTorr}$. Polarizing frequency: $14380.4 \mathrm{MHz}$. Sample interval $20 \mathrm{~ns} .8 \cdot 10^{6}$ averaging cycles. b) Rotational transition $19 F_{1}(4)-F_{2}(3)$ of $^{74} \mathrm{GeH}_{4}$. Cell temperature: $20^{\circ} \mathrm{C}$. Gas pressure: $4.3 \mathrm{~m}$ Torr. Polarizing frequency: $12690.5 \mathrm{MHz}$. Sample interval $20 \mathrm{~ns} .5^{5} \cdot 10^{6}$ averaging cycles. c) Rotational transition $20 F_{1}(4)-F_{2}(2)$ of ${ }^{74} \mathrm{GeH}_{4}$. Cell temperature: $20^{\circ} \mathrm{C}$. Gas pressure: $4.1 \mathrm{mTorr}$. Polarizing frequency: $17026.8 \mathrm{MHz}$. Sample interval $20 \mathrm{~ns} .5 \cdot 10^{6}$ averaging cycles.

with

$$
\begin{aligned}
A= & (1 / 2)\left(h_{1}^{\mathrm{u}}-h_{1}^{1}\right)\left(C^{\prime}-C^{\prime \prime}\right), \\
B= & (3 / 4)\left(h_{2}^{\mathrm{u}}-h_{2}^{1}\right) \\
& \cdot\left[C^{\prime}\left(C^{\prime}+1\right)-C^{\prime \prime}\left(C^{\prime \prime}+1\right)\right] /[J(J+1)] .
\end{aligned}
$$

In order to calculate the constants $c_{d}$ and $d$ we selected the best resolved transition $19 F_{2}(4)-19 F_{1}(2)$ (Figure $1 \mathrm{a}$ ). The values of $A$ and $B$ as well as the measured splittings are given in Table 1 . The resulting constants are $c_{d}=10.07(53) \mathrm{kHz}$ and $d=8.9(55) \mathrm{kHz}$. The errors are estimated from the precision of the frequency determination of the multiplet components. The values of Tab. 1 were used to calculate the splittings of all measured transitions, which were unsplit or partly split. Always the pattern agreed. A
Table 1. Numerical values of $A$ and $B$ and corresponding measured splittings $\Delta(\Delta v)$ for the $19 F_{2}(4)-19 F_{1}(2)$. All data are given in $\mathrm{kHz}$. For line frequencies see Figure 1.

\begin{tabular}{llrl}
\hline$F^{\prime \prime}-F^{\prime}$ & \multicolumn{1}{l}{$A$} & \multicolumn{1}{l}{$B$} & $\Delta(\Delta v)$ \\
\hline $18-19$ & 7.28634 & 0.743431 & 80 \\
$19-20$ & 7.66984 & -0.706212 & 71 \\
\hline
\end{tabular}

second possibility is to assume $d$ to be fixed at its theoretical value $d=7.88 \mathrm{kHz}$ [5]. In this case we obtain $c_{d}=10.08(55) \mathrm{kHz}$ (mean value). This result may be compared with the spin-rotation constant $c_{d}=5.5(50) \mathrm{kHz}$ measured by magnetic resonance methods [5]. 


\section{Conclusion}

Today MWFT spectroscopy seems to be a well suited method to determine spin-rotation and spin-spin interaction constants of tetrahedral molecules with high accuracy in comparison to magnetic resonance methods. The ground state spectra of methane and silane have been also investigated $[11,12]$ but a hyperfine structure has not yet been reported.

[1] W. Stahl, H. Dreizler, L. Jörissen, and W. A. Kreiner, Z. Naturforsch. 41 a, 747 (1986)

[2] I. Merke, Diplomarbeit, Kiel 1986.

[3] R. F. Curl, Jr., J. V. V. Kasper, and K. S. Pitzer, J. Chem. Phys. 46, 3220 (1966).

[4] P.-N. Yi, I. Ozier, and C. H. Anderson, Phys. Rev. 165, 92 (1967).

[5] I. Ozier, L. M. Crapo, and S. S. Lee, Phys. Rev. 172, 63 (1968).

[6] I. Ozier, P.-N. Yi, A. Khosla, and N. F. Ramsey, Phys. Rev. Lett. 24, 642 (1970).

\section{Acknowledgement}

The authors wish to thank Jon T. Hougen from the National Bureau of Standards for his helpful advices. We further thank the members of the Kiel microwave group for help and discussions, the Deutsche Forschungsgemeinschaft and Fonds der Chemie for funds.

[7] J. T. Hougen, J. Mol. Spectrosc. 46, 490 (1973).

[8] F. Michelot, B. Bobin and J. Moret-Bailly, J. Mol. Spectrosc, 76, 374 (1979).

[9] F. Michelot, J. Mol. Spectrosc, 106, 77 (1984).

[10] A. J. Dorney and J. K. J. Watson, J. Mol. Spectrosc. 42, 135 (1972).

[11] M. Oldani, M. Andrist, and A. Bauder, and A. G. Robiette, J. Mol. Spectrosc, 110, 93 (1985).

[12] M. Oldani, A. Bauder, and A. G. Robiette, J. Mol. Spectrosc. 117, 60 (1986). 